were not using hormonal contraception. We used Poisson mixed effects models to determine if genital HSV DNA detection or lesion frequency differed throughout the menstrual cycle, categorised into four seven-day phases based on most proximate first day of menstrual bleeding: early and late for each of follicular and luteal.

Results In 189 women aged 19-46 (median age 33) who collected 9307 genital swabs, HSV was detected on 1822 days (20\%). The rate of shedding was $21 \%$ during the early follicular phase versus $18 \%$ during late luteal $(\mathrm{RR}=1.2,95 \% \mathrm{CI}$ $1.0-1.4, \mathrm{p}=0.04), 21 \%$ during late follicular $(\mathrm{RR}=1.2$ relative to late luteal, 95\% CI 1.0-1.5, $\mathrm{p}=0.06$ ), and $19 \%$ during early luteal (RR 1.1 relative to late luteal, 95\% CI $0.9-1.3$, $\mathrm{p}=0.53$ ). In sensitivity analyses reducing misclassification of phase by excluding samples $>10$ days from day 1 of menses, these observations were strengthened. The pattern was similar for genital lesions, present on $13 \%$ of days during the follicular phase and 11\% during the luteal phase.

Conclusion In women with established HSV infection, genital HSV-2 shedding and lesions were slightly more common during the early follicular phase of the menstrual cycle than in the luteal phase. These cyclic variations may be related to changes in oestrogen and progesterone affecting vaginal immunity.

\section{P2.15 PERSISTENCE OF CYSTOISOSPORA BELLI IN HIV PATIENTS: DRUG FAILURE, RESISTANCE OF THE PARASITE OR INCOMPLETE IMMUNE RESTORATION?}

${ }^{1}$ Failoc-Rojas Virgilio E, ${ }^{2}$ Hernández-Córdova Gustavo, ${ }^{3}$ Silva-Díaz Heber, ${ }^{3}$ FernándezValverde Darwin. 'Universidad Nacional Pedro Ruiz Gallo, Lambayeque - Peru; ' Universidad Peruana Cayetano Heredia, Lima - Peru; ${ }^{3}$ Laboratorio De Parasitología, Metaxénicas Y Zoonosis, Hospital Regional Lambayeque

\subsection{6/sextrans-2017-053264.192}

Introduction: Cystoisospora belli infection is one of the most important causes of watery diarrhoea in patients with HIV and causes high rates of morbidity and mortality. The introduction of highly active antiretroviral therapy (HAART) in recent years has improved the ability of immune response and decreased viral load.

Methods A prospective study was performed among HIV patients admitted to hospital of Lambayeque. Herein we describe seven clinical cases of diarrhoea caused by $C$. belli infection in HIV patients, who showed different evolution and response to treatment.

Results Five were males, with a mean age of 32 years and chronic diarrhoea. Four patients had recurrent diarrhoea despite receiving secondary prophylaxis with cotrimoxazole and good viral and immunological response to HAART in addition to specific treatment. While others were not receiving HAART and prophylaxis, but responded well to treatment.

Conclusion: C. belli is an important cause of diarrhoea in HIV patients on HAART and prophylaxis. In this study, 7 cases of patients with HIV infection and diarrhoea caused by C. belli are presented. Three of those were newly diagnosed, so they did not receive HAART and secondary cotrimoxazole prophylaxis and their CD4+ levels were below 200/uL. However, they responded favourably to C. belli treatment, with no recurrences. Meanwhile, the other four patients were receiving HAART, secondary prophylaxis and had evidence of immune restoration $(>200 \mathrm{CD} 4+/ \mathrm{uL})$, but the standard treatment failed to eradicate the parasite. This clinical contradiction has been reported previously with some particularities.

We suggest that persistent infection may be due to drug failure by intrinsic or extrinsic to the parasite causes, or to defects in restoration of the intestinal immune system, or both.

\section{P2.16 OBSTETRIC AND PERINATAL OUTCOMES IN PREGNANT WOMEN WITH PERINATALLY ACQUIRED HIV-INFECTION -PRELIMINARY RESULTS}

${ }^{1}$ Gilmar de ${ }^{1}$ Souza Osmundo Junior, ${ }^{1}$ Agatha Sacramento Rodrigues, ${ }^{2}$ Vera Lucia Jornada Krebs, ${ }^{1}$ Marcelo Zugaib Rossana Pulcineli Vieira Francisco. ${ }^{1}$ Department of Obstetrics and Gynaecology, São Paulo University Medical School, São Paulo, Brazil; ${ }^{2}$ Department of Neonatology, São Paulo University Medical School, São Paulo, Brazil

\subsection{6/sextrans-2017-053264.193}

Introduction Prevalence of pregnancy in women with perinatally acquired HIV infection (PAH-W) has markedly increased. HIV infection is related to preterm delivery, small for gestational age fetus (SGA), and pre-eclampsia (PE). PAH-W have higher incidence of longer periods of HIV-infection, lower levels of CD4 cells, and higher viral loads (VL). Available data are inconclusive about $\mathrm{PAH}-\mathrm{W}$ related risks on pregnancy. The present study aims to analyse obstetric and perinatal outcomes in PAH-W.

Methods Retrospective cohort study involving pregnant PAHW followed from 2005 to 2015. Results were compared to those obtained from pregnant women with sexually transmitted HIV-infection (STH-W). Antiretroviral therapy (ART), vertical transmission rate, obstetric and perinatal outcomes were considered. Chi-square, Fish extract, Mann-Whitney-Wilcoxon, and Student's $\mathrm{T}$ tests were applied to non-continuous and continuous samples.

Results PAH-W group consisted of 14 patients and STH-W group had 17 women. PAH-W were younger (20.1 3.0 vs. $29.9 \pm 7.7$ years, $p=0.001$ ) than STH-W. Nevertheless, groups were similar regarding to CD4 counts $(471.7 \pm 271$ vs. 302.7 \pm 183.5 cells $/ \mathrm{mm} 3, \mathrm{p}=0.21$ ), proportion of undetectable 34 thweek VL (41.7\% amongst the case group vs. $66.7 \%, p=0.26)$, and to the 34 th-week VL levels $(2.9 \pm 0.8$ vs. $2.7 \pm 0.9 \log$, $\mathrm{p}=0,68)$. Prevalence of SGA (3 in PAH-W group vs. 1 in STH-W, $\mathrm{p}=0.28$ ) and preterm labour (0 in PAH-W group vs. 2 in control group, $\mathrm{p}=0.49$ ) were also similar in both groups. Neither cases of PE and spontaneous preterm delivery nor HIV-infected infant were found.

Conclusion PAH-W and STH-W had similar obstetric and perinatal results. Since both groups were comparable in CD4 counts and 34th-week VL levels, it is possible the occurrence of negative obstetric outcomes may be more likely related to the severity of HIV infection than to the mechanism of infection itself. Larger studies are still necessary to determine the role of PAH-W in pregnancy. However PAH-W should be emphasised on adequate ART and achieving low VL levels in order to reduce their obstetric and perinatal risks.

\section{P2.17 ABSTRACT WITHDRAWN}

